\title{
Leadless pacemaker and cremation
}

\author{
Raghav Bhargava, ${ }^{1}$ Balram Bhargava ${ }^{2}$
}

'Department of Acute Medicine, Royal Shrewsbury Hospital, Shrewsbury, UK 2Department of Cardiology, AllMS, New Delhi, India

\section{Correspondence to} Dr Raghav Bhargava, Department of Acute Medicine Royal Shrewsbury Hospital, Mytton Oak Road, Shrewsbury SY3 8XQ, UK;

raghavbhargava90@gmail.com
Implantable cardiac pacemakers save lives and improve quality of life. Each year about a million pacemakers are implanted worldwide, a quarter of them in the USA. Over the past 50 years several innovations have taken place in the miniaturisation of the pacemaker including circuitry, battery and lead systems. These advances have increased generator lifespan, improved programmability and simplified the 'implant procedure. ${ }^{1}$

The non-surgical, leadless pacemaker is an innovative example of miniaturisation. Normal pacemaker batteries explode when exposed to very high temperatures and need to be explanted at the time of cremation. Miniaturisation and dramatic developments have taken place in battery development, but the explosive potential of the battery during cremation remains a conundrum and possibly increases with smaller more energy-dense batteries. The cremation hazard of this percutaneous pacemaker has been poorly studied, but with the rising number of cremations the world over, this hazard remains a serious concern. ${ }^{2} 3$

Several potential complications related to the transvenous lead (infection, venous obstruction, insulation break and conduction break), the subcutaneous pulse generator (pocket infection, haematoma) and the procedure itself (pneumothorax, tamponade) have led to innovations in the development of a self-contained leadless pacemaker. Although the concept is not new, it has taken over 40 years for two companies to develop this device. Medtronic Inc (Minneapolis, Minnesota, USA) developed their 'Micra' transcatheter pacing system about the size of a vitamin pill and claim it is the world's smallest pacemaker; St Jude Medical (St Paul, Minnesota, USA) developed and tested the 'Nanostim' leadless pacemaker, which received a CE mark in 2013. ${ }^{4}$ Both these pacemakers are leadless, inserted through the femoral vein and anchored entirely inside the right ventricular apex. There is no subcutaneous pocket or scar and the device cannot be explanted easily once endothelialised ( 3 months). These pacemakers are being implanted all over Europe, but remain under investigation in the USA. ${ }^{2}$ Since they are single-chamber pacemakers and do not offer physiological dual-chamber pacing, they are expected to replace $20-30 \%$ of the current implants and perhaps more in developing countries.

The pacemaker battery has presented challenges to obtaining high levels of safety and reliability. The early implanted pacemakers used a nickel cadmium or zinc mercury battery. These batteries had very short lifespans. The lithium iodine battery invented in 1972 by Wilson Greatbatch (inventor of the first implantable pacemaker) remains the most acceptable power source for cardiac pacemakers because of its adaptable shape, minimal weight, corrosion resistance and excellent current drain characteristics, marked safety and predictable long life. ${ }^{1}$ Lithium batteries with liquid cathode systems are not suitable for pacemakers, but are suitable for automatic external defibrillators. Lithium batteries with solid cathode systems have slow self-discharge characteristics and are suitable for memory backup in watches, cameras and calculators. The solid electrolyte lithium cells are characterised by long service life at low drain currents even at high temperatures and are ideal for pacemakers. The lithium iodine-polyvinylpyridine is the principal cardiac pacemaker battery today. The leadless pacemaker battery is lithium carbon monofluoride or lithium polycarbon fluoride and offers even higher energy density. ${ }^{5}$ It is hypothesized that the more energy dense a battery is, the greater is the risk of explosion. ${ }^{6}$

Cremation rates have increased greatly worldwide and vary widely across the world, with Japan having a rate $>95 \%$ while in other countries-for example, Italy and Poland, the rate is $<10 \%$. Factors influencing cremation include culture and religion-for example, the cremation rate in Muslim, Eastern Orthodox and Roman Catholic majority countries is much lower owing to religious attitudes to cremation. Almost everyone adhering to Hinduism, Sikhism, Buddhism and Jainism cremate their dead, making India one of the countries with the highest rate of cremation of around $85 \%$.

Cremation rates in the major commonwealth countries UK, Canada, Australia and New Zealand have risen dramatically to around $70 \%$. The cremation rate in the USA has been increasing steadily with the national average rising from 4\% in 1960 to $40 \%$ in 2010 . Data suggest that about $5 \%$ of bodies undergoing cremation contain metallic objects. $^{7}$

Cremation takes place in the primary combustion chamber, which is initially heated to $700^{\circ} \mathrm{C}$ for about $1 \mathrm{~h}$, followed by combustion of the body at higher temperatures of $900-1100^{\circ} \mathrm{C}$ for about $2 \mathrm{~h}^{8}$ The remains are subsequently cooled in a secondary chamber. Most metal prostheses are not fully combusted at these temperatures, and as a result the Environment Protection Act 1990 (UK) has mandated the temperatures of all crematoria to be raised to $1600^{\circ} \mathrm{C}$ to minimise emissions of dioxin. The first reported pacemaker explosion in 1976 led to damage of the brickwork lining of the cremation chamber. This explosion was attributed to the hydrogen produced by the mercury zinc batteries. It is further, suggested that current lithium batteries when heated to high temperatures are even more explosive. Every implanted battery should pass a very stringent 'cremation test' of withstanding $1300^{\circ} \mathrm{C}$ for $90 \mathrm{~min}$. Until it becomes law, it is 


\section{Viewpoints}

mandatory to sign cremation forms and the coroner's form clearly stating the presence and adequate removal of the pacemaker from the corpse before cremation. More recently, handheld metal detectors have been tested and shown to be highly sensitive for detecting metal prostheses, including pacemakers, before cremation. ${ }^{9}$ Further, it is against the code of practice of crematoria staff to open sealed coffins and they rely on accurate information provided by these forms. Despite this, a study found that about $50 \%$ of the crematoria in the UK have had pacemaker explosions. These explosions lead to losses due to damage of the crematorium and injury to crematoria staff; it is therefore the legal responsibility of physicians, funeral directors and health authorities to prevent such accidents. ${ }^{10} 11$

The leadless pacemaker design makes it more difficult to detect as well as remove. These pacemakers are much smaller and have greater energy-dense batteries with possibly a higher potential for explosion. One solution to this problem is to make simulation studies publicly available to show the safety of these devices at such high temperatures. These will have an important bearing in obtaining US Food and Drug Administration approval. Their presence will not be detected clinically or with hand-held metal detectors and will be difficult to explant after death. Since routine pacemakers are implanted subcutaneously, they can easily be removed by a small incision before cremation. For removal of the leadless pacemaker a complete autopsy will be required with opening of the chest and the heart before cremation and may be socially unacceptable. Even if they are found to be safe for cremation, the social, cultural, legal and universal acceptability of these devices for cremation will take time.

Contributors The article was conceived and written by RB. BB gave guidance for the final preparation of the manuscript.

Competing interests None declared.

Provenance and peer review Not commissioned; externally peer reviewed.

\section{REFERENCES}

1 Beck $H$, Boden WE, Patibandla $S$, et al. 50th Anniversary of the first successful permanent pacemaker implantation in the United States: historical review and future directions. Am J Cardiol 2010;106:810-18.

2 Reddy VY, Knops RE, Sperzel J, et al. Permanent leadless cardiac pacing: results of the LEADLESS trial. Circulation 2014;129:1466-71.

3 Gale CP, Mulley GP. Pacemaker explosions in crematoria: problems and possible solutions. J R Soc Med 2002;95:353-5.

4 Spickler JW, Rasor NS, Kezdi P, et al. Totally self-contained intracardiac pacemaker. J Electrocardiol 1970;3:325-31.

5 Mallela VS, Ilankumaran V, Rao NS. Trends in cardiac pacemaker batteries. Indian Pacing Electrophysiol J 2004:4:201-12.

6 http://www.technologyreview.com/view/419767/new-ultra-battery-as-energy-denseas-high-explosives/

7 http://en.wikipedia.org/wiki/List_of_countries_by_cremation_rate

8 Holm KF, Hjortshøj S, Pehrson $\bar{S}_{\text {, et }}$ al. Implanted cardiac devices are reliably detected by commercially available metal detectors. Scand Cardiovasc J 2013:47:271-4

9 Barry M. Metal residues after cremation. BMJ 1994;308:390.

10 Ghosh D, James MA, Palmer RB. Electrical injury on removal of implantable defibrillator after death. Heart 1997;77:484-5.

11 Gale CP, Mulley GP. A migrating pacemaker. Postgrad Med J 2005;81:198-9. 